\title{
Enhancing the Security of Fiber Optical Network using Cryptography
}

doi : https://doi.org/10.32628/IJSRCSEIT

\section{Sheenu Sachdeva ${ }^{1}$, Dinesh ${ }^{2}$}

${ }^{1}$ Assistant Professor, Department of CSE, JCDM College of Engineering, Sirsa

${ }^{2}$ M.Tech. Scholar, Department of CSE, JCDM College of Engineering, Sirsa

\begin{abstract}
Article Info

Volume 7, Issue 5

Page Number: 115-119

Publication Issue :

September-October-2021

\section{Article History}

Accepted : 15 Oct 2021

Published : 30 Oct 2021

An optical fiber is an important communication channel as it proposes a high bandwidth and less attenuation, and can be easy challenging assistances such as huge-quality data transmission and others in computer networks. The basic optical transmission system consists of three basic elements which are fiber media (transmission channel), light sources as the input (covert electric signal into optic signal) and light detector as the output (convert optic signal into electric signal). FBG is the key component in optical communication system as, dispersion compensators, filters and flatteners gain. There is need to introduce mechanism that should be capable to provide data security in less time. More over there is need to introduce energy efficient mechanism that should be capable to reduce the size of packet during data transmission. There are several problems that are faced due to attenuation in fiber optics. There is need of repeater in order to regenerate the signals. Process working principle of proposed technique that is being used enhancement of security in steganography has been discussed with process flow and result. It states how brute force and timing attack are not applicable on proposed technique.

Keywords : Optisystem, fiber optic, data security, RSA Encryption algorithm
\end{abstract}

\section{INTRODUCTION}

Fiber is an integral part of modern day data communication infrastructure and can be found along roads, in buildings, industries, hospitals and machinery. The fiber itself is a strand of silica based glass and its dimensions similar to those of a human hair that are surrounded by a transparent cladding. Light can be transmitted along the fiber over long distances at very high data rates providing an ideal medium for the transport of information. Fiber optic communication is a method of transmitting information from one place to another by sending light through an optical fiber. The basic optical transmission system consists of three basic elements which are fiber media (transmission channel), light sources as the input (covert electric signal into optic signal) and light detector as the output (convert optic signal into electric signal).

Moreover several problems are faced due to attenuation in fiber optics. There is need of repeater in order to regenerate the signals. The attenuation in fiber optics has been influenced byzincrease in 
distance, number of joints and number of connectors. More over there is need to introduce energy efficient mechanism that should be capable to reduce the size of packet during data transmission. The major issue considered in research is data security. The different encryption mechanism used in previous research makes the process very slow. There is need to introduce mechanism that should be capable to provide data security in less time. Process working principle of proposed technique that is being used enhancement of security in steganography has been discussed with process flow and result. It states how brute force and timing attack are not applicable on proposed technique.

\section{LITERATURE REVIEW}

In 2019 Y. Dong et al. [9] Effect of tapered fiber structure on the PbS Nanomaterials optical fiber amplifier excited by evanescent wave. PbS nanomaterials fiber amplifier was fabricated by coating $\mathrm{PbS}$ nanomaterials onto tapered fiber. $\mathrm{PbS}$ is deposited by atomic layer deposition technique. The influence to optical properties is discussed when changing the structure of tapered fiber.

In 2019 L. Huang et al.[2] Fabrication and sensing characteristics of $2 \mu \mathrm{m}$ long period fiber grating written in double cladding fiber by $\mathrm{CO} 2$ laser. They demonstrate the fabrication of long period fiber gratings (LPFGs) in double cladding fiber in midinfrared wavelength. The experimental results show that the LPFGs with a resonance dip in mid-infrared wavelength have much higher sensitivity.

In 2018 R. P. M. Julie et al.[3] A Novel High Resolution Optical Time Delay Cable Measurement System Utilized in Fiber Verification in Radio Interferometry. A long distance optical fiber cable measurement is described that delivers $\sim 0.2$ picosecond (ps) delay resolution with capability for high accuracy relative distance extraction over 10's of microseconds of delay.

In 2018 J. Wen et al.[4] All-Fiber OAM Amplifier With High Purity and Broadband Spectrum Gain Based on Fused Taper Vortex-Beam Coupler. All-fiber orbital angular momentum (OAM) amplifier with high purity and broadband spectrum gain based on a fused taper vortex-beam coupler (VBC) has been studied. Active and passive few-mode fibers (FMFs), which have similar geometric parameters, are fabricated. A VBC using a single-mode fiber and a passive FMF is designed and fabricated.

In 2017 L. Ding et al.[5] Fiber optic sensor based on polarization-dependent absorption of graphene. Graphene has been reported to show polarizationdependent optical absorption in the visible spectral range, that it shows more absorption for s-polarized light than p-polarized light. They study further on it and demonstrate that this unique property can be utilized to design a fiber optic sensor, which works in a similar way of surface plasmon resonance (SPR) fiber optic sensor.

\section{RESEARCH METHODOLOGY}

Research Methodology stands for a pattern or method which is followed in a research by the researchers. It can be define as a process which is considered in order to make research on a particular topic. There is different type of research methodologies which should be known by a researcher before he stated his research work.

Establishment of fiber network in order to provide energy efficient solution. Reduce the size of content in order to minimize the size of packet and reduce packet dropping probability. Establishment of security mechanism that should be less time consuming as compare to previous encryption technique. Establishment of sender and receiver module and performing data transmission over fiber 
network by encrypting data using xor mechanism after compressing it. Comparing the performance of proposed work to existing research in order to prove the quality of proposed work.

\section{OBJECTIVE OF RESEARCH}

Several objectives are there that are considered in the research work. Such are listed below:

1. To study the existing researches of data encryption in fiber based network environment

2. To review the traditional transmission and protocol for security in network environment

3. To consider the influencing security factor, transmission media and protocol used for transmission

4. To use the fiber optic to improve the energy efficiency and minimize the Attenuation during secure data transmission.

5. To prove the proposed work better as compare to the traditional protocol for data security and energy efficiency.

\section{RESULTS}

\subsection{SENDER SIDE IMPLEMENTATION}

When Sender is ready for the Communication and transmission starts at that time the design view at the sender side and the important information for communication is required is shown in the below screenshot.

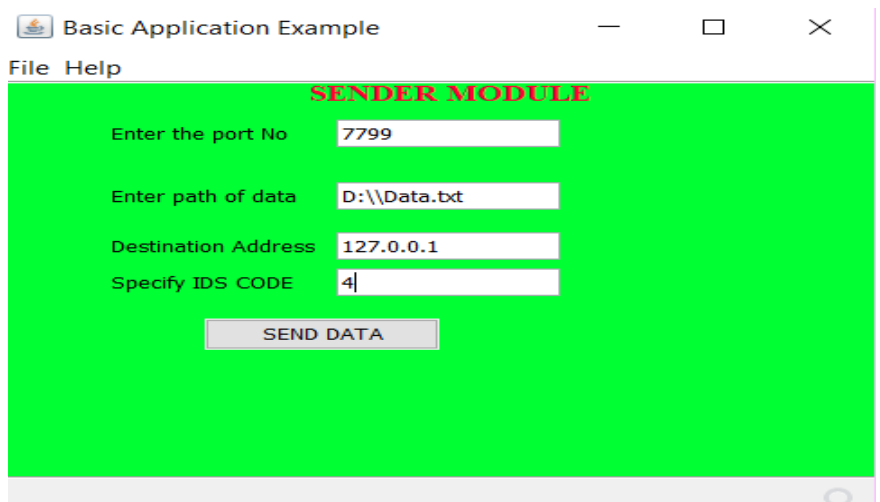

FIGURE 4.1 DESIGN VIEW OF SENDER SIDE WITH IDS CODE AS OTP
Figure 4.1 is showing that after specifying the path of the file which is going to transfer we need to specify an IDS code as OTP so that receiver should pair with the sender for getting the file at the receiver end. After put the IDS sender is now enabled to send data. and when we click on SEND DATA data is transferred to the receiver end.

\subsection{RECIEVER SIDE IMPLEMENTATION}

When Receiver is ready for the Communication and transmission comes to the end. There is a design view at the Receiver side so that the important information comes at right place is required is shown in the below screenshot.

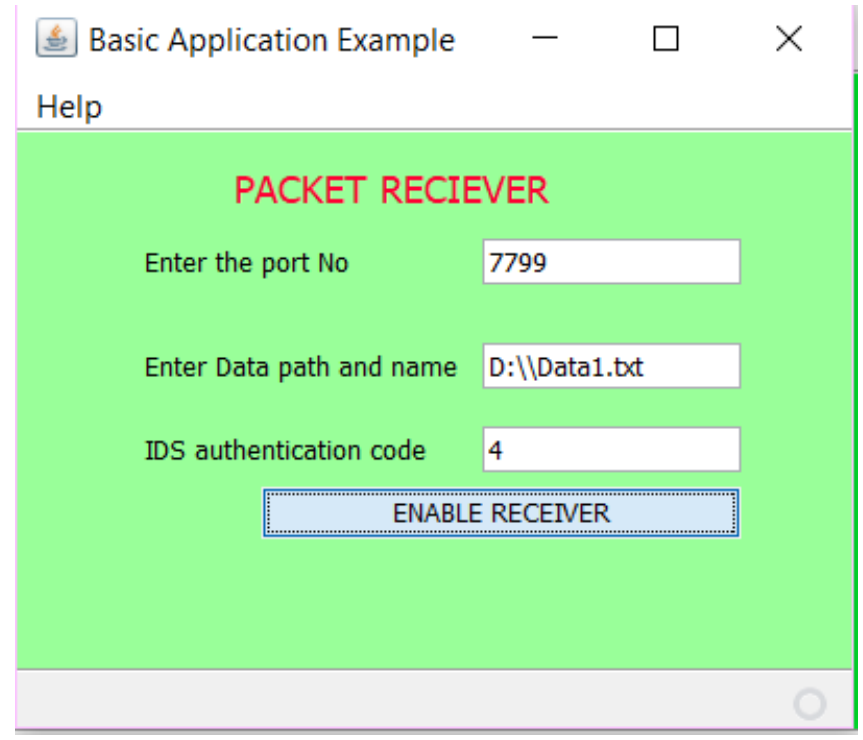

FIGURE 4.2 DESIGN VIEW OF RECEIVER SIDE with IDS as OTP

Figure 4.2 is showing the design view of the receiver application. In this application during execution of client/receiver side module, with the requirement of port number 7799 and the full file path with its name and extension, there is also a need for IDS as OTP number as same as sender side for authorization to receive the file from the sender. After put the IDS number now when we click on ENABLE RECEIVER option file is showed at the receiver drive. The 
receiver should be enabled first then the sender performs its action after a click on send data.

\subsection{TESTING PROCESS}

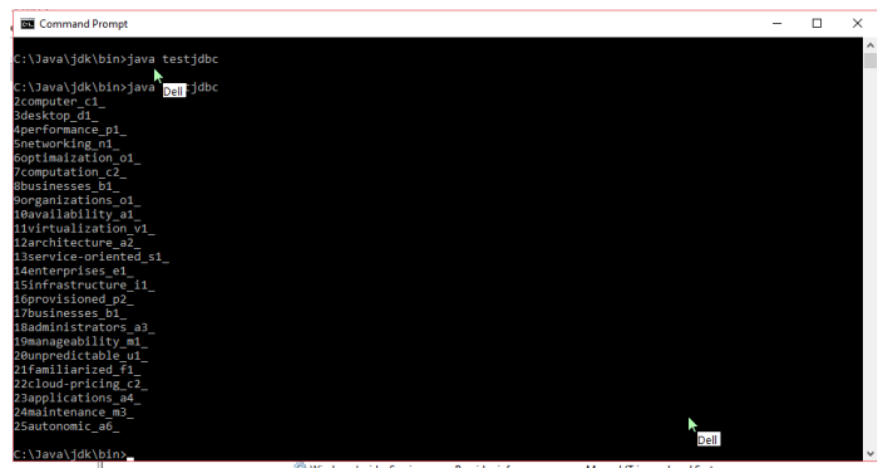

\subsection{REPLACEMENT ALGORITHM}

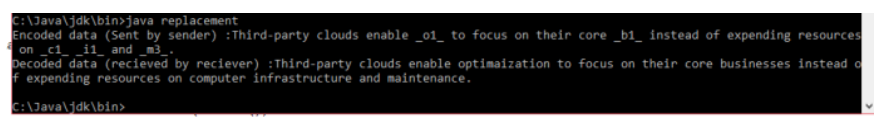

FIGURE 4.10 PROCESS OF REPLACEMENT ALGORITHM

Figure 4.10 shows the output Encryption process of the proposed model. In this figure, data has been sending in an encoded form rather than in text format. This encoded data cannot be accessible by unauthorized users and nearly impossible for a user to decrypt data without the algorithm keys. After performing the testing and compression process the Multiplicative encryption algorithm and advanced encryption standard (AES) algorithm are applied to the Text file.

\section{CONCLUSION}

After considering existing research, need for data security came in demand. It was found that the probability of cyber attacks is more in case of existing research. Moreover there was a need to increase the performance of network during data transmission. Simulation represents that security to data has been increased using a multilayer encryption mechanism. The proposed work is found capable of increasing the security of the network by providing a more secure and efficient mechanism. Packets are compressed and reduced in order to travel over network without congestion issues. Such research would play a significant role in network application. Content of network applications would be made more secure and capable to travel at long distances without dropping.

\section{REFERENCES}

[1]. B. C. Yao et al., "Partially reduced graphene oxide based FRET on fiber-optic interferometer for biochemical detection," 2017 25th Optical Fiber Sensors Conference (OFS), Jeju, 2017, pp. 1-4, doi: 10.1117/12.2263182.

[2]. L. Huang, C. Jiang, X. Zhao and Y. Liu, "Fabrication and sensing characteristics of $2 \mu \mathrm{m}$ long period fiber grating written in double cladding fiber by CO2 laser," 2019 18th International Conference on Optical Communications and Networks (ICOCN), Huangshan, China, 2019, pp. 1-2, doi: 10.1109/ICOCN.2019.8934831.

[3]. R. P. M. Julie, T. D. Abbott, J. P. Burger and R. Siebrits, "A Novel High Resolution Optical Time Delay Cable Measurement System Utilized in Fiber Verification in Radio Interferometry," 2018 IEEE International Frequency Control Symposium (IFCS), Olympic Valley, CA, 2018, pp. 1-4, doi: 10.1109/FCS.2018.8597450.

[4]. J. Wen et al., "All-Fiber OAM Amplifier With High Purity and Broadband Spectrum Gain Based on Fused Taper Vortex-Beam Coupler," in IEEE Photonics Journal, vol. 10, no. 6, pp. 1-8, Dec. 2018, Art no. 7105308, doi: 10.1109/JPHOT.2018.2872040.

[5]. L. Ding, C. Xu, Z. Xia, B. Xu and J. Huang, "Fiber optic sensor based on polarizationdependent absorption of graphene," 2017 25th Optical Fiber Sensors Conference (OFS), Jeju, 2017, pp. 1-4, doi: 10.1117/12.2265346.

[6]. D. Yang, D. Li, J. Tao, Y. Fang, X. Mao and W. Tong, "An optical fiber comprehensive analysis system for spectral-attenuation and geometry 
Sheenu Sachdeva et al Int. J. Sci. Res. Comput. Sci. Eng. Inf. Technol, September-October-2021, 7 (5) : 115-119

parameters measurement," 2017 Conference on Lasers and Electro-Optics Pacific Rim (CLEOPR), Singapore, 2017, pp. 1-2, doi: 10.1109/CLEOPR.2017.8118861.

[7]. P. Barcik, P. Munster, P. Dejdar, T. Horvath and J. Vojtech, "Measurement of Polarization Transient Effects Caused by Mechanical Stress on Optical Fiber," 2019 International Workshop on Fiber Optics in Access Networks (FOAN), Sarajevo, Bosnia and Herzegovina, 2019, pp. 2628, doi: 10.1109/FOAN.2019.8933658.

\section{Cite this article as :}

Sheenu Sachdeva, Dinesh, "Enhancing the Security of Fiber Optical Network using Cryptography", International Journal of Scientific Research in Computer Science, Engineering and Information Technology (IJSRCSEIT), ISSN : 2456-3307, Volume 7 Issue 5, pp. 115-119, September-October 2021.

Journal URL : https://ijsrcseit.com/CSEIT217541 | 